\title{
CARACTERIZAÇÃO DE RESÍDUOS SÓLIDOS DA BARRAGEM DE REJEITOS DA MINA DO SOSSEGO (CANA ̃̃ DOS CARAJÁS-PA)
}

\author{
J. C. NASCIMENTO ${ }^{1}$, H. C. CORDEIRO ${ }^{2}$, N. F. A. F. CORAL ${ }^{3}$, J. A. M CORRÊA ${ }^{3}$, S. G. C. \\ MOREIRA $^{4}$, E. M. S. RODRIGUES ${ }^{2}$ \\ ${ }^{1}$ Universidade Federal do Pará, Instituto de Tecnologia, Faculdade de Engenharia Química \\ ${ }^{2}$ Universidade Federal do Pará, Instituto de Ciências Exatas e Naturais, Faculdade de Química \\ ${ }^{3}$ Universidade Federal do Pará, Instituto de Geociências, Programa de Pós Graduação em Geologia e \\ Geoquímica. \\ ${ }^{4}$ Universidade Federal do Pará, Instituto de Ciências Exatas e Naturais, Departamento de Física \\ E-mail para contato: juliane.nascimento@itec.ufpa.br
}

\begin{abstract}
RESUMO - Barragens de Rejeitos são estruturas que têm por finalidade reter resíduos sólidos e água oriundos do processo de beneficiamento de minério. Os rejeitos apresentam na sua composição partículas dissolvidas e em suspensão, além de metais pesados e reagentes químicos. Este trabalho tem por objetivo caracterizar resíduos do processamento de cobre presentes na Barragem de Rejeitos da Mina do Sossego (Canaã dos Carajás-PA). Foram selecionados cinco pontos de coleta (sedimento e água), os quais foram caracterizados por DRX, MEV/EDS e Raman, além da análise de $\mathrm{pH}$. As amostras de sedimento são basicamente constituídas por Actinolita $\left(\mathrm{Ca}_{2}(\mathrm{Mg}, \mathrm{Fe})_{5} \mathrm{Si}_{8} \mathrm{O}_{22}(\mathrm{OH})\right)$, Albita $\left(\mathrm{Na}\left(\mathrm{AlSi}_{3} \mathrm{O}_{8}\right)\right)$, Biotita $\left(\left(\mathrm{K}_{1.71} \mathrm{Na}_{0.22} \mathrm{Ba}_{0.07}\right)\left(\mathrm{Mg}_{3.4} \mathrm{Fe}_{1.5} \mathrm{Ti}_{0.98}\right)\left(\mathrm{Al}_{1.52} \mathrm{Si}_{6.48} \mathrm{O}_{20}\right) \mathrm{O}_{2.28}(\mathrm{OH})_{1.72}\right)$, Clinocloro $\left(\left(\mathrm{Mg}_{2.96} \mathrm{Fe}_{1.55} \mathrm{Fe}_{.136} \mathrm{Al}_{1.275}\right)\left(\mathrm{Si}_{2.622} \mathrm{Al}_{1.376} \mathrm{O}_{10}\right)(\mathrm{OH})_{8}\right)$ e Quartzo $\left(\mathrm{SiO}_{2}\right)$. As amostras de água apresentaram pH entre 6,0 e 7,0. Esses resultados preliminares são promissores, pois os íons presentes nos sedimentos os qualificam como material de partida para a síntese de adsorventes químicos e/ou catalisadores heterogêneos.
\end{abstract}

\section{INTRODUÇÃO}

No Estado do Pará, jazem enormes riquezas minerais que lhe concedem uma clara vocação mineradora e a grande possibilidade de tornar-se o maior produtor de minérios do país nos próximos dez anos. Têm-se conhecimento da existência de imensas reservas de minério de ferro de alta qualidade, bem como de cobre e de ouro localizadas na Província Mineral de Carajás, entretanto, seus teores foram completamente mensurados (Setran, 2009).

O processo de beneficiamento desses minérios produzem resíduos sólidos/líquidos que possuem como característica elevado grau de toxicidade, além de partículas dissolvidas e em suspensão, metais pesados e reagentes, representando uma importante fonte de poluição, que pode acontecer através de drenagem ácida, infiltração dos contaminantes para o lençol freático, contaminação do solo e água superficial a jusante (Lozano, 2006; Duarte, 2008). 
Uma alternativa a disposição desordenada desses resíduos no ambiente é a sua estocagem em barragens de contenção, permitindo o controle adequado de toda a água percolante, para com isso garantir requisitos de controle da poluição, segurança, economia, e capacidade limite de armazenamento (Passos, 2009).

Atualmente, as companhias de mineração promovem o estudo de práticas que equilibrem a exploração mineral e o meio ambiente, por meio de ações mitigadoras dos danos ao meio ambiente. As estratégias mais inteligentes e compensadoras são aquelas que aproveitam os rejeitos/resíduos, valorizam os subprodutos e neutralizam as poluições com os resíduos da própria mineração ou com produtos locais. Na China, por exemplo, os rejeitos de minério de Ferro são utilizados em materiais de construção, na fabricação de artefatos cerâmicos e para fornecer elementos traços aos fertilizantes. (Wolff, 2009; Zhang et al., 2006).

Assim, esse trabalho tem como foco principal a caracterização dos rejeitos, oriundos do beneficiamento do minério de cobre (Mina do Sossego-Canaã dos Carajás), a fim de que possam ser utilizados como material de partida na síntese de novos materiais, os quais serão empregados como adsorventes químicos (metais e efluentes industriais) e/ou catalisadores heterogêneos.

\section{MATERIAIS E MÉTODOS}

Foram selecionadas amostras de sedimentos (S1, S3, S5, S7 e S9) e água (A1, A3, A5, A7 e A9), dispostas em cinco pontos ao longo da Barragem de Rejeitos. A coleta iniciou-se próxima a descarga da usina e foi finalizada na central de abastecimento de água da usina (Fig. 01) (Tab. 01). Essa coleta foi realizada em novembro de 2013. Essas amostras foram comparadas diretamente com amostras coletadas em uma etapa de campo preliminar, ocorrida em outubro de 2012, de modo a verificar se as características físicas e químicas modificaram de acordo com a estação. Na primeira coleta estava ocorrendo a transição entre as estações seca e chuvosa, enquanto que na segunda, estava ocorrendo um excesso de chuvas (RTCIII-03A, RTCIII-03B, RTCIII-03C, RTCIII-03D, RTCIII03E).

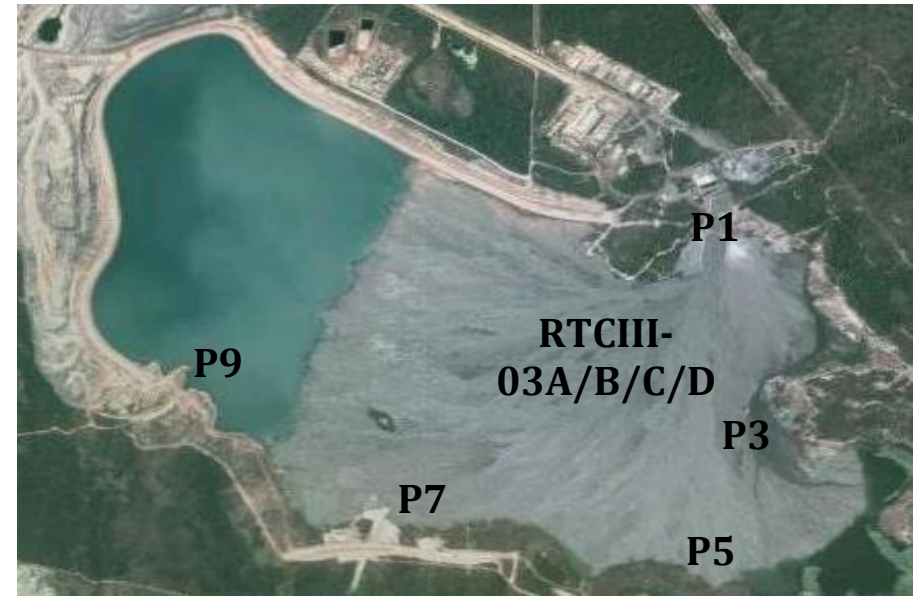

Figura 01- Barragem de Rejeitos. Mina Sossego, Canaã dos Carajás. Fonte: Google Maps
Tabela 01 - Geoposicionamento das amostras

\begin{tabular}{c|c|c}
\hline & S & W \\
\hline P1 & $06^{\circ} 27^{\prime} 076^{\prime}$, & $050^{\circ} 04^{\prime} 449^{\prime \prime}$ \\
\hline P3 & $06^{\circ} 27^{\prime}$ & $050^{\circ} 05^{\prime} 084^{\prime \prime}$ \\
\hline P5 & $06^{\circ} 27^{\prime} 16.8^{\prime \prime}$ & $050^{\circ} 05^{\prime} 57.5^{\prime}$ \\
\hline P7 & $06^{\circ} 26^{\prime} 14.5^{\prime}$, & $050^{\circ} 05^{\prime} 41.6^{\prime \prime}$ \\
\hline P9 & $06^{\circ} 25^{\prime} 49.11^{\prime}$, & $050^{\circ} 05^{\prime} 7.5^{\prime}$ \\
\hline RTCIII-03A & & \\
RTCIII-03B & $06^{\circ} 27^{\prime} 16,8^{\prime}$, & $050^{\circ} 04^{\prime} 50,8^{\prime}$, \\
RTCIII-03C \\
RTCIII-03D
\end{tabular}


A caracterização desses sedimentos foi realizada por meio das técnicas de Difração de Raios-X (DRX), Microscopia Eletrônica de Varredura (MEV/EDS), Espectroscopia Raman, enquanto que na água foi realizada apenas a análise de $\mathrm{pH}$. As condições analíticas utilizadas foram as seguintes, DRX: X PERT-PRO (PW 3050 PAN analytical), software HighScore 2.0, $\mathrm{CoKa}(\lambda=1,78897)$, monocromadores de níquel (Ni), $2 \theta$ de $5-70^{\circ}, 2^{\circ} / \mathrm{min}$, $8 \mathrm{~s}$ e fenda $1 / 8,40 \mathrm{~mA}$ e $40 \mathrm{kV}$; MEV/EDS: HITACHI, TM 3000, $15 \mathrm{kV}$, com software para análise line scan, no modo eletrônico retroespalhados e metalização Au; Espectroscopia Raman: Jobin Ivon, $633 \mathrm{~nm} \mathrm{2w} \mathrm{-}$ Nd: YAG, 0 - 3800 cm-1 , 10 blocos de 10 s; Papel de pH (0-14) Macherey-Nagel.

\section{RESULTADOS E DISCUSSÃO}

\subsection{DRX}

Os dados de DRX indicaram uma composição mineralógica muito similar para as amostras S1, S3 e S7: clinocloro e albita como minerais principais e actinolita, biotita e quartzo como minerais secundários. As amostras S5 e S9 apresentaram quartzo como mineral principal e albita e espangolita como minerais secundários. A amostra S5 também apresentou o clinocloro, assim como a amostra S9 também apresentou como mineral principal a biotita, e como mineral secundário a actinolita (Fig. 02/ Tab. 02).

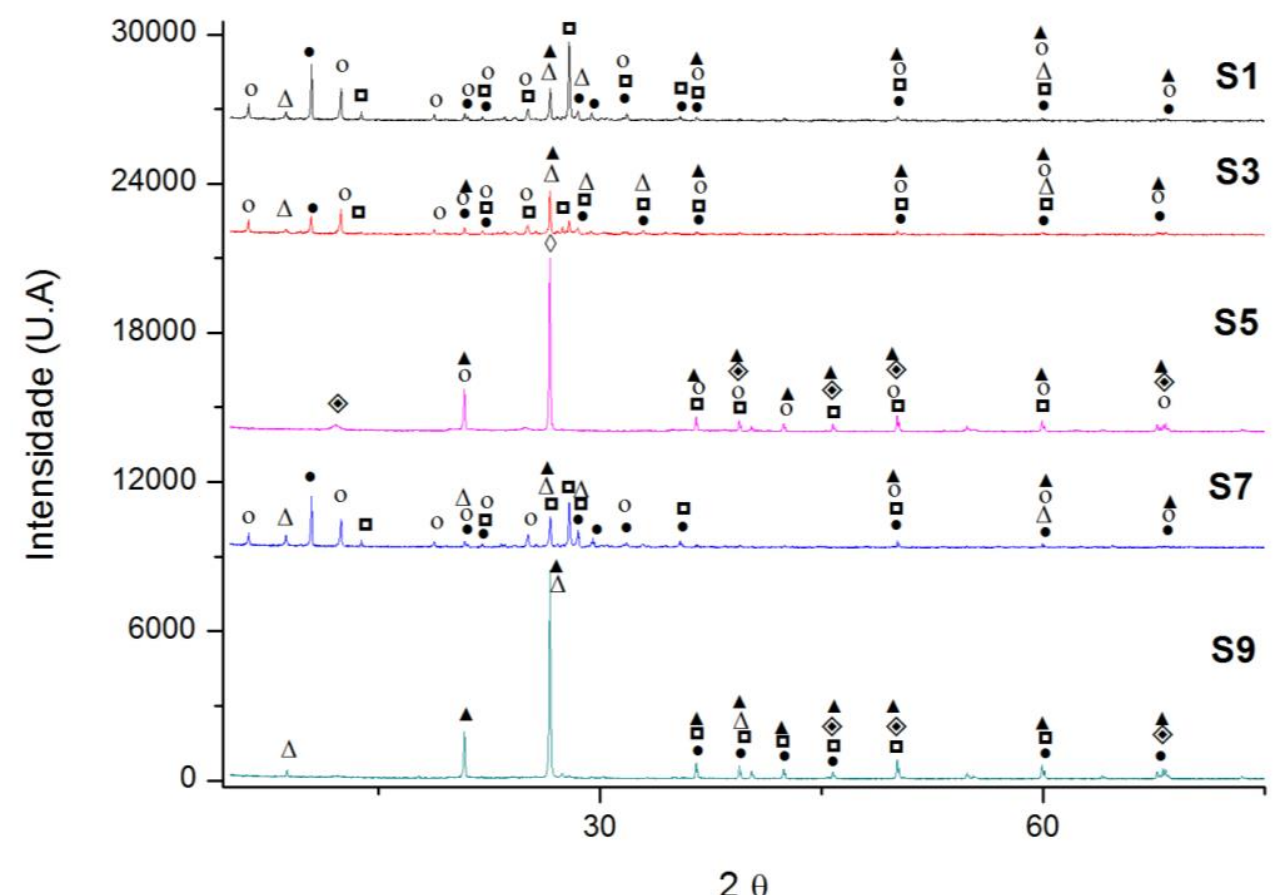

Figura 02 - Difratogramas das amostras S1, S3, S5, S7 e S9 (Legenda: •-Actinolita, - -Albita, $\Delta$-Biotita, oClinocloro, $\diamond$-Espangolita, $\mathbf{\Lambda}$-Quartzo). 
Tabela 02 - Teor (\% p/p) estimado por DRX da composição mineralógica das amostras S1, S3, S5, S7 e S9.

\begin{tabular}{|c|c|c|c|c|c|}
\hline Minerais & S1 & S3 & S5 & S7 & S9 \\
\hline $\begin{array}{c}\text { Actinolita } \\
\mathrm{Ca}_{2}(\mathrm{Mg}, \mathrm{Fe})_{5} \mathrm{Si}_{8} \mathrm{O}_{22}(\mathrm{OH})\end{array}$ & 19 & 19 & --- & 20,2 & 10,9 \\
\hline $\begin{array}{c}\text { Albita } \\
\mathrm{Na}\left(\mathrm{AlSi}_{3} \mathrm{O}_{8}\right) \\
\end{array}$ & 27 & 19 & 31,3 & 27,3 & 7,9 \\
\hline $\begin{array}{c}\text { Biotita } \\
\left(\mathrm{K}_{1.71} \mathrm{Na}_{0.22} \mathrm{Ba}_{0.07}\right)\left(\mathrm{Mg}_{3.4} \mathrm{Fe}_{1.5} \mathrm{Ti}_{0.98}\right) \\
\left(\mathrm{Al}_{1.52} \mathrm{Si}_{6.48} \mathrm{O}_{20}\right) \mathrm{O}_{2.28}(\mathrm{OH})_{1.72}\end{array}$ & 14 & 17 & --- & 13,1 & 49,5 \\
\hline $\begin{array}{c}\text { Clinocloro } \\
\left(\mathrm{Mg}_{2.96} \mathrm{Fe}_{1.55} \mathrm{Fe}_{.136} \mathrm{Al}_{1.275}\right) \\
\left(\mathrm{Si}_{2.622} \mathrm{Al}_{1.376} \mathrm{O}_{10}\right)(\mathrm{OH})_{8}\end{array}$ & 29 & 33 & 19,2 & 26,3 & --- \\
\hline $\begin{array}{c}\text { Espangolita } \\
\mathrm{Cu}_{6}\left(\mathrm{Al}_{0.85} \mathrm{Fe}_{0.15}\right)\left(\mathrm{SO}_{4}\right)(\mathrm{OH})_{12} \\
\left(\mathrm{Cl}_{0.635}(\mathrm{OH})_{0.365}\right)\left(\mathrm{H}_{2} \mathrm{O}\right)_{3} \\
\end{array}$ & --- & --- & 3 & --- & 1 \\
\hline $\begin{array}{c}\text { Quartzo } \\
\mathrm{SiO}_{2}\end{array}$ & 11 & 12 & 46,5 & 13,1 & 30,7 \\
\hline
\end{tabular}

De maneira geral, não foi observada alteração expressiva na composição mineralógica das amostras conforme as mesmas se afastavam progressivamente do local de descarga da usina. As amostras apresentaram apenas pequenas diferenças quanto aos minerais principais e secundários. Como exceção, tem-se a amostra S9 que apresentou um alto teor de biotita, quando comparada com as demais amostras, sendo justificada por ter sido coletada no ponto mais afastado do despejo dos resíduos do processo de mineração. "A priori” os resíduos apresentaram uma dispersão pouco variável ao longo da barragem.

Os dados provenientes da coleta anterior, todas localizadas as proximidades do local de descarga da usina, indicaram uma composição mineralógica para as amostras RTCIII-03A e RTCIII03D: albita e quartzo como minerais principais e actinolita, biotita e clinocloro como minerais secundários; sendo também na RTCIII-03D encontrado o mineral magnetita. Já as amostras RTCIII03B e RTCIII-03C apresentaram biotita e quartzo como minerais principais e actinolita, albita, clinocloro e marialita como minerais secundários (Fig. 03/Tab. 03) (Lima et al., 2013). 


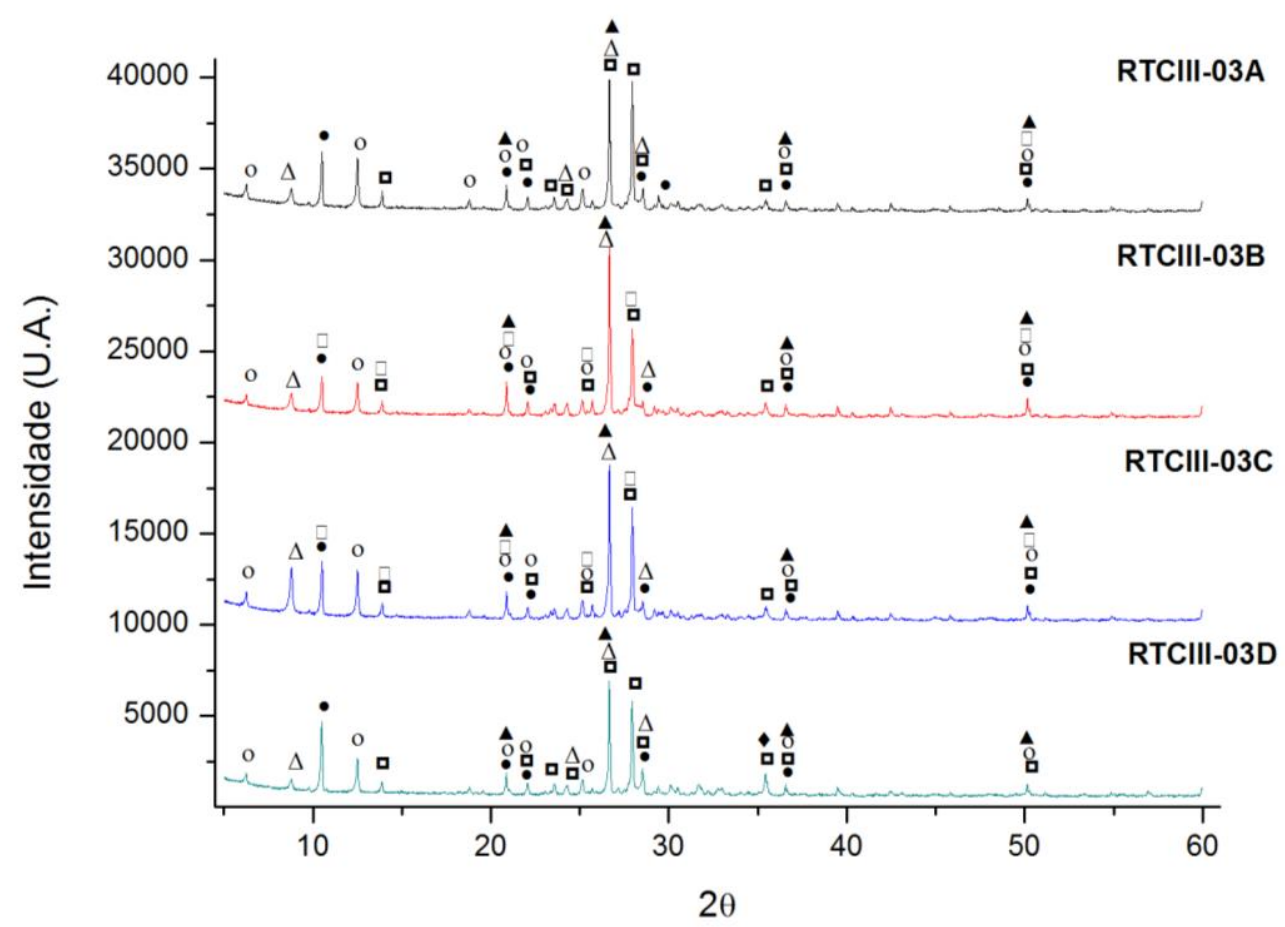

Figura 03 - Difratogramas das amostras RTCIII-03A, RTCIII-03B, RTCIII-03C, RTCIII-03D (Legenda:

Actinolita, $\boldsymbol{\square}$-Albita, $\Delta$-Biotita, o-Clinocloro, $\bullet$ - Magnetita, $\square$ Marialita, $\boldsymbol{\Delta}$-Quartzo).

Tabela 03 - Teor (\% p/p) estimado por DRX da composição mineralógica das amostras RTCIII-03A, RTCIII03B, RTCIII-03C e RTCIII-03D.

\begin{tabular}{|c|c|c|c|c|}
\hline Minerais & RTCIII-03A & RTCIII-03B & RTCIII-03C & RTCIII-03D \\
\hline $\begin{array}{c}\text { Actinolita } \\
\mathrm{Ca}_{2}(\mathrm{Mg}, \mathrm{Fe})_{5} \mathrm{Si}_{8} \mathrm{O}_{22}(\mathrm{OH})\end{array}$ & 9 & 8,9 & 9 & 14,1 \\
\hline $\begin{array}{c}\text { Albita } \\
\mathrm{Na}\left(\mathrm{AlSi}_{3} \mathrm{O}_{8}\right)\end{array}$ & 36 & 17,8 & 17 & 26,3 \\
\hline $\begin{array}{c}\text { Biotita } \\
\left(\mathrm{K}_{1.71} \mathrm{Na}_{0.22} \mathrm{Ba}_{0.07}\right)\left(\mathrm{Mg}_{3.4} \mathrm{Fe}_{1.5} \mathrm{Ti}_{0.98}\right) \\
\left(\mathrm{Al}_{1.52} \mathrm{Si}_{6.48} \mathrm{O}_{20}\right) \mathrm{O}_{2.28}(\mathrm{OH})_{1.72}\end{array}$ & 16 & 20,8 & 24 & 14,1 \\
\hline $\begin{array}{c}\text { Clinocloro } \\
\left(\mathrm{Mg}_{2.96} \mathrm{Fe}_{1.55} \mathrm{Fe}_{.136} \mathrm{Al}_{1.275}\right) \\
\left(\mathrm{Si}_{2.622} \mathrm{Al}_{1.376} \mathrm{O}_{10}\right)(\mathrm{OH})_{8}\end{array}$ & 14 & 11,9 & 14 & 12,1 \\
\hline $\begin{array}{c}\text { Magnetita } \\
\mathrm{Fe}_{3} \mathrm{O}_{4}\end{array}$ & --- & --- & --- & 7,1 \\
\hline $\begin{array}{c}\text { Marialita } \\
\mathrm{Na}_{4}\left(\mathrm{Al}_{3} \mathrm{Si}_{9} \mathrm{O}_{24}\right) \mathrm{Cl}\end{array}$ & --- & 5 & 5 & --- \\
\hline $\begin{array}{c}\text { Quartzo } \\
\mathrm{SiO}_{2}\end{array}$ & 25 & 35,6 & 31 & 26,3 \\
\hline
\end{tabular}

A composição mineralógica dos rejeitos, coletados nas duas etapas, permaneceu quase inalterada, variando somente nas proporções dos minerais presentes, bem como na presença de uma pequena quantidade de marialita nas amostras RTCIII-03B e RTCIII-03C. 


\subsection{MEV/EDS}

As análises por MEV/EDS confirmaram a identificação de fases minerais encontradas no DRX.

A albita, mineral encontrado em todas as amostras, apresenta-se de forma anédrica e possui cristais com dimensões em torno de $0,539 \mathrm{~mm}$. Sua composição química $(\% \mathrm{p} / \mathrm{p})$ mostra-se em semelhança com a obtida para a fórmula mínima $\left(\mathrm{Na}\left(\mathrm{AlSi}_{3} \mathrm{O}_{8}\right)\right): 48,812 \% \mathrm{O}, 8,767 \% \mathrm{Na}, 10,290 \%$ Al, 32,132\% Si (Fig. 04a/ Tab. 04).

O clinocloro, encontrado em todas as amostras com exceção da S9, apresentam-se subédricos e possui cristais com dimensões em torno de $0,0692 \mathrm{~mm}$. Sua composição química (\%p/p) mostra-se análoga com a obtida para a fórmula mínima $\left(\left(\mathrm{Mg}_{2.96} \mathrm{Fe}_{1.55} \mathrm{Fe}_{.136} \mathrm{Al}_{1.275}\right)\left(\mathrm{Si}_{2.622} \mathrm{Al}_{1.376} \mathrm{O}_{10}\right)(\mathrm{OH})_{8}\right)$ : $47,420 \% \mathrm{O}, 11,846 \% \mathrm{Mg}, 11,778 \% \mathrm{Al}, 12,125 \% \mathrm{Si}, 15,503 \% \mathrm{Fe}, 1,328 \% \mathrm{H}$ (Fig. 04b/ Tab. 04).

O quartzo, encontrado em todas as amostras, apresentam-se anédricos e possui cristais com dimensões em torno de $0,515 \mathrm{~mm}$. Sua composição química mostra-se de acordo com a obtida para a fórmula mínima $\left(\mathrm{SiO}_{2}\right)$ : 53,257\% O, 46,743\% Si (Fig. 04c/ Tab. 04).
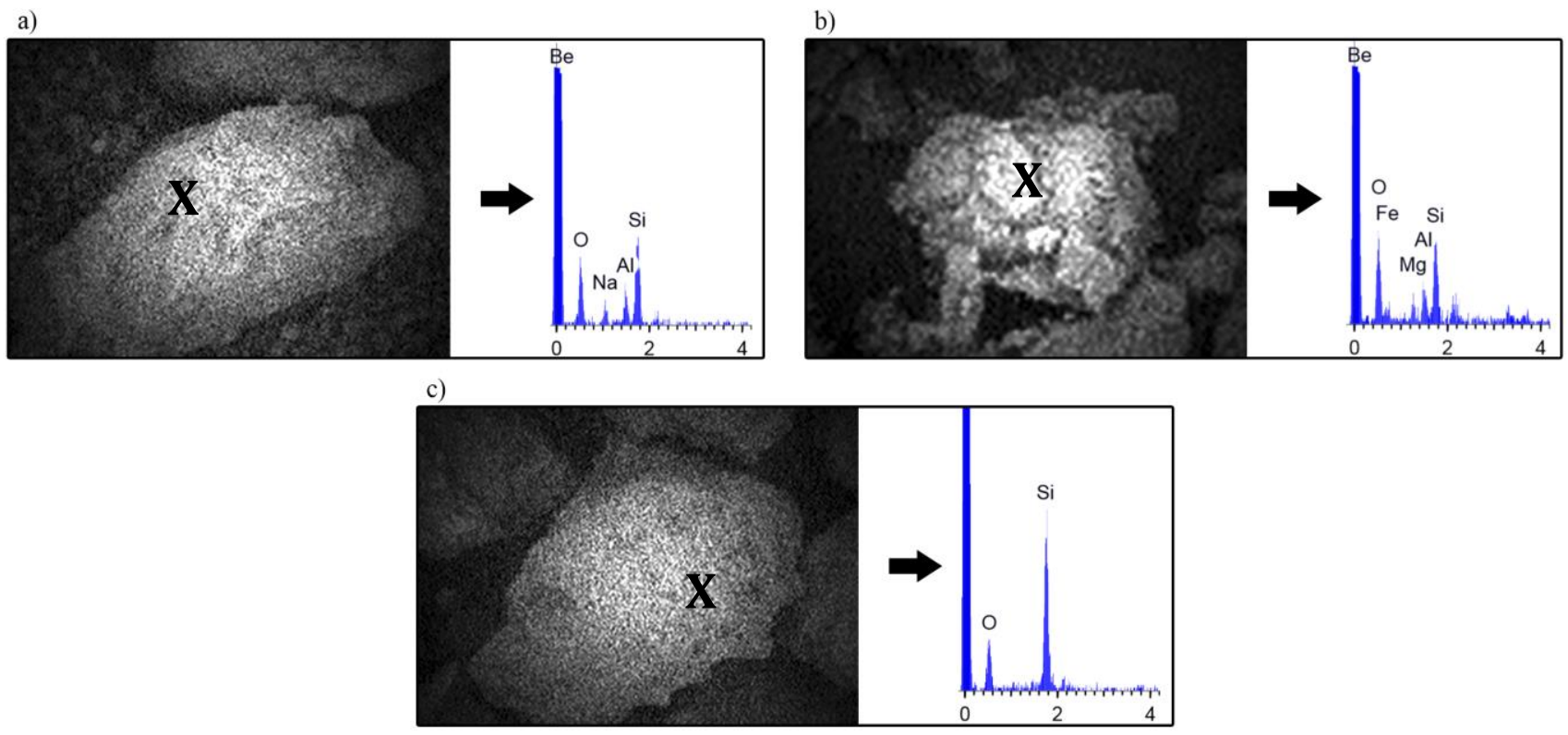

Figura 04 - Micrografias MEV/EDS e seus respectivos espectros eletromagnéticos: a) Mineral albita na amostra S1; b) Mineral clinocloro na amostra S7; c) Mineral quartzo na amostra S1.

Tabela 04 - Composição química estimada (\%p/p) dos minerais albita, clinocloro e quartzo, visualizada nas amostras $\mathrm{S} 1, \mathrm{~S} 7$ e S1, respectivamente.

\begin{tabular}{c|c|c|c|c|c|c}
\hline \multirow{2}{*}{ Minerais } & \multicolumn{7}{|c}{ Elementos } \\
\cline { 2 - 7 } & $\mathrm{O}$ & $\mathrm{Na}$ & $\mathrm{Mg}$ & $\mathrm{Al}$ & $\mathrm{Si}$ & $\mathrm{Fe}$ \\
\hline Albita & 58,110 & 8,729 & --- & 8,556 & 24,606 & --- \\
\hline Clinocloro & 53,550 & --- & 5,194 & 6,820 & 18,720 & 15,716 \\
\hline Quartzo & 59,150 & --- & --- & --- & 40,50 & --- \\
\hline
\end{tabular}




\subsection{Espectroscopia Raman}

A análise por Espectroscopia Raman permitiu a identificação de bandas características dos minerais albita e quartzo (Fig. 05/Tab.05/Tab.06).

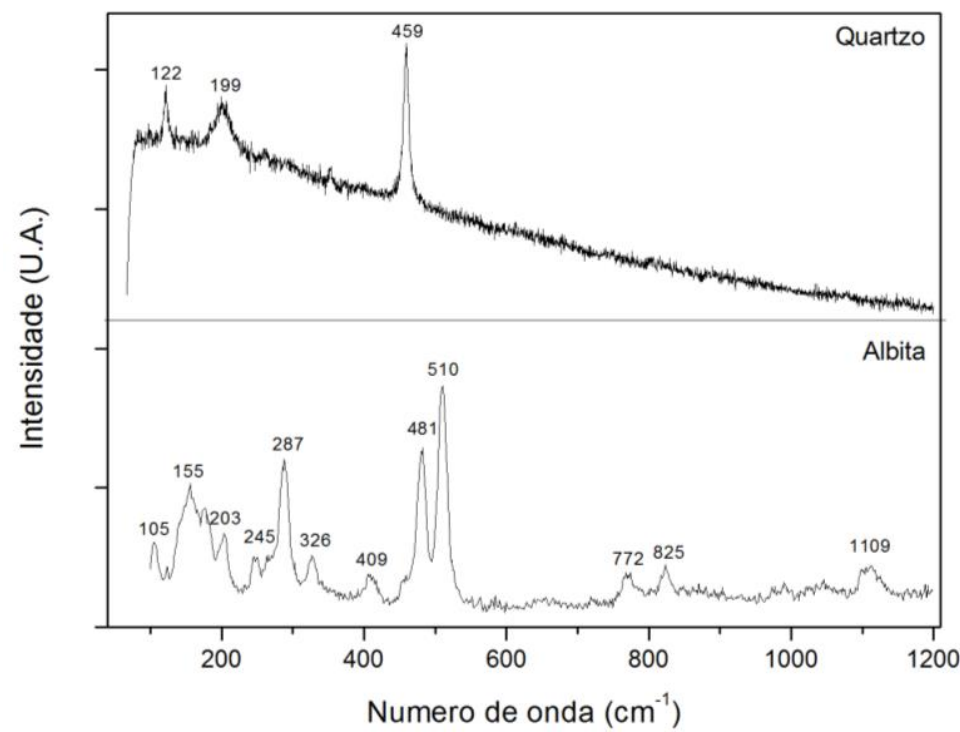

Figura 05 - Espectro Raman do mineral quartzo (amostra S5) e do do mineral albita (amostra S7).
Tabela 05 - Bandas referentes ao espectro do mineral quartzo. (Ling, et

\begin{tabular}{c|c}
\hline $\mathrm{N}^{\circ}$ de onda & Descrição das bandas \\
\hline 122 & $\delta(\mathrm{Si}-\mathrm{O}-\mathrm{Si})$ \\
\hline 199 & $\delta(\mathrm{Si}-\mathrm{O}-\mathrm{Si})$ \\
\hline 459 & $\delta(\mathrm{O}-\mathrm{Si}-\mathrm{O})$ \\
\hline
\end{tabular}

Tabela 06 - Bandas referentes ao espectro do mineral albita (Mckeown, 2005)

\begin{tabular}{c|c}
\hline $\mathrm{N}^{\mathbf{o}}$ de onda & Descrição das bandas \\
\hline 105 & Rede \\
\hline 155 & Rede \\
\hline 409 & $\delta(\mathrm{O}-\mathrm{Al})$ \\
\hline 481 & $\mathrm{v}(\mathrm{Na}-\mathrm{O})$ \\
\hline 510 & $\delta(\mathrm{O}-\mathrm{Na}-\mathrm{O})$ \\
\hline 772 & $\delta(\mathrm{Si}-\mathrm{O}-\mathrm{Si}), \mathrm{v}(\mathrm{Na}-\mathrm{O})$ \\
\hline 825 & $\delta(\mathrm{Si}-\mathrm{O}-\mathrm{Si}), \mathrm{v}(\mathrm{Na}-\mathrm{O})$ \\
\hline 1109 & $v(\mathrm{Al}-\mathrm{O})$ \\
\hline
\end{tabular}

\subsection{Estudo de pH}

A medição "in loco" do pH das amostras de água ao longo da barragem de rejeitos, indicou valores na faixa de 6,0/7,0 para todas as amostras. Não observou-se variação em decorrência do afastamento da descarga da usina. O resultado foi comparado com o de uma coleta anterior (RTCIII03E), que apresentou $\mathrm{pH}$ de 5,5. Essa variação de 5,5 a 7,0 registrada de um ano para o outro, é explicada, provavelmente, pela primeira coleta ter sido realizada em outubro de 2012, quando estava ocorrendo a transição das estações seca e chuvosa, enquanto que a segunda coleta, realizada em novembro de 2013 ocorreu no período onde houve excesso de chuvas (Tab.07) (Melo, et al., 2013).

Tabela $07-\mathrm{pH}$ das amostras de água coletadas ao longo da barragem de rejeitos.

\begin{tabular}{c|c|c|c|c|c}
\hline A1 & A3 & A5 & A7 & A9 & RTCIII-03E \\
\hline 7,0 & 7,0 & 6,0 & 7,0 & 6,0 & 5,5 \\
\hline
\end{tabular}

\section{CONCLUSÃO}

A composição mineralógica das amostras estudadas: actinolita $\left(\mathrm{Ca}_{2}(\mathrm{Mg}, \mathrm{Fe})_{5} \mathrm{Si}_{8} \mathrm{O}_{22}(\mathrm{OH})\right)$, albita $\left(\mathrm{Na}\left(\mathrm{AlSi}_{3} \mathrm{O}_{8}\right)\right)$, biotita $\left(\left(\mathrm{K}_{1.71} \mathrm{Na}_{0.22} \mathrm{Ba}_{0.07}\right)\left(\mathrm{Mg}_{3.4} \mathrm{Fe}_{1.5} \mathrm{Ti}_{0.98}\right)\left(\mathrm{A}_{11.52} \mathrm{Si}_{6.48} \mathrm{O}_{20}\right) \mathrm{O}_{2.28}(\mathrm{OH})_{1.72}\right)$, clinocloro $\left(\left(\mathrm{Mg}_{2.96} \mathrm{Fe}_{1.55} \mathrm{Fe}_{.136} \mathrm{~A}_{11.275}\right)\left(\mathrm{Si}_{2.622} \mathrm{~A}_{11.376} \mathrm{O}_{10}\right)(\mathrm{OH})_{8}\right)$ e quartzo $\left(\mathrm{SiO}_{2}\right)$ é bastante promissor no sentido de 


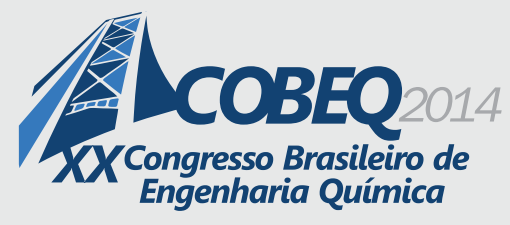

19 a 22 de outubro de 2014
Florianópolis/SC

utilizar os resíduos sólidos da exploração do minério de cobre da Mina do Sossego (Canaã dos Carajás/Pa) como material de partida para a síntese de hidróxidos duplo lamelares, uma vez que cátions bi/trivalentes $\left(\mathrm{K}^{+}, \mathrm{Na}^{+}, \mathrm{Si}^{2+}, \mathrm{Mg}^{2+}, \mathrm{Ba}^{2+}, \mathrm{Ca}^{2+}, \mathrm{Fe}^{2+}, \mathrm{Al}^{3+}\right.$ e Si$\left.{ }^{4+}\right)$ bem como ânions $\left(\mathrm{Cl}^{-}, \mathrm{OH}^{-}\right)$ estão presentes, além de contribuir significativamente para amenizar parte dos danos ambientais que a mineração ocasiona.

\section{REFERÊNCIAS}

DUARTE, A. P. Classificação das barragens de contenção de rejeitos de mineração e de resíduos industriais no estado de Minas Gerais em relação ao potencial de risco. 114p. Dissertação (Mestrado em Saneamento, Meio Ambiente e Recursos Hídricos) - Universidade Federal de Minas Gerais, Belo Horizonte, 2008.

GOOGLE MAPS. Disponível em <https://www.google.com.br/maps/@-6.440945,$50.079718,6326 \mathrm{~m} /$ data=!3m1!1e3>. Acesso em 07 abr. de 2014.

LIMA, L. S.; NASCIMENTO, J. C.; RODRIGUES, J.; CORAL, N. F. A. F.; PEREIRA, P. M.; RODRIGUES, E. M. S.; MENDES, A. S. Caracterização de rejeitos de minério cobre (mina do sossego - canaã dos carajás - sudoeste do pará). In: ENCONTRO DE PROFISSIONAIS DA QUÍMICA DA AMAZÔNIA, 13, 2013. Belém.

LING, Z. C.; WANG, A.; JOLLIFF, B. L., LI, C.; LIU, J.; BIAN, W.; REN, X.; MU, L.; SU, Y. Raman spectroscopic study of quartz in lunar soils from apollo 14 and 15 missions. 40TH LUNAR AND PLANETARY SCIENCE CONFERENCE, 2009. Texas.

LOZANO, F. A. E. Seleção de locais para barragens de rejeitos usando o método de análise hierárquica. 128p. Dissertação (Mestrado em Engenharia) - Escola politécnica da Universidade de São Paulo, São Paulo, 2006.

MCKEOWN, D. A. Raman spectroscopy and vibrational analyses of albite: From $25^{\circ} \mathrm{C}$ through the melting temperature. American Mineralogist, v. 90, p. 1506-1517, 2005.

MELO, A. C. M; FRASSONI, A; SISMANOGL, R. A; Nobre, P; SELUCHI, M. Persistem condições de neutralidade no oceano pacífico equatorial. Infoclima: Boletim de informações climáticas do CPETEC/INPE. São Paulo, Ano. 20, n. 11, 26 nov. 2013. Disponível em: <http://infoclima.cptec.inpe.br/>. Acesso em: 12 abr. 2014

PASSOS, N. C. S. T. Barragem de Rejeito: Avaliação dos parâmetros geotécnicos de rejeitos de minério de ferro utilizando ensaios de campo - Um estudo de caso. 79p. Trabalho de Conclusão de Curso (Graduação) - Universidade Federal do Paraná, Curitiba, 2009.

SECRETARIA DE ESTADO DE TRANSPORTES DO ESTADO DO PARÁ. Análise Espacial da Mineração no Pará. p. 2 - 12. São Paulo, 2009.

WOLFF, A. P. Caracterização de Rejeitos de Minério de Ferro de Minas da Vale. 90p. Dissertação (Mestrado em Engenharia Mineral) - Universidade Federal de Ouro Preto, Minas Gerais, 2009.

ZHANG, S.; XUE, X., DUAN, P., et al. (2006). Current situation and comprehensive utilization of iron one tailings resources. Journal of mining Science. V.42, n.4, p.403-407. 